Check for updates

Cite this: Sustainable Energy Fuels, 2020, 4, 254

Received 4th September 2019 Accepted 3rd November 2019

DOI: $10.1039 / c 9 s e 00750 d$

rsc.li/sustainable-energy

\section{Minimising the levelised cost of electricity for bifacial solar panel arrays using Bayesian optimisation $\uparrow$}

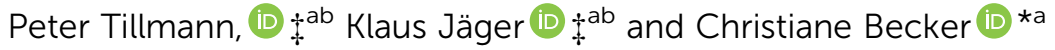

Bifacial solar module technology is a quickly growing market in the photovoltaics (PV) sector. By utilising light impinging on both, front and back sides of the module, actual limitations of conventional monofacial solar modules can be overcome at almost no additional costs. Optimising large-scale bifacial solar power plants with regard to minimum levelised cost of electricity (LCOE), however, is challenging due to the vast amount of free parameters such as module inclination angle and distance, module and land costs, character of the surroundings, weather conditions and geographic position. We present a detailed illumination model for bifacial PV modules in a large PV field and calculate the annual energy yield exemplary for four locations with different climates. By applying the Bayesian optimisation algorithm we determine the global minimum of the LCOE for bifacial and monofacial PV fields at these two locations considering land costs in the model. We find that currently established design guidelines for mono- and bifacial solar farms often do not yield the minimum LCOE. Our algorithm finds solar panel configurations yielding up to $23 \%$ lower LCOE compared to the established configuration with the module tilt angle equal to the latitude and the module distance chosen such that no mutual shading of neighboring solar panels occurs at winter solstice. Our algorithm enables the user to extract clear design guidelines for mono- and bifacial large-scale solar power plants for most regions on Earth and further accelerates the development of competitively viable photovoltaic systems.
\end{abstract}

\section{Introduction}

The record power conversion efficiency (PCE) of monofacial silicon solar cells - currently the dominant solar-cell technology - is $26.7 \%{ }^{1}$ and approaches the physical limit of around $29.4 \%$, which was calculated by Richter et $a .^{2}$ Photovoltaic (PV) systems consisting of bifacial solar modules can generate a significantly higher annual energy yield (EY) than systems using conventional monofacial PV modules, because bifacial solar modules not only utilize light impinging onto their front, but also illumination onto their rear side., ${ }^{3,4}$ Furthermore, advanced solarcell concepts such as PERC, PERT, PERL (passivated emitter rear contact/totally-diffused/locally-diffused) and IBC (interdigitated back contact) can easily be manufactured as bifacial solar cells. ${ }^{5}$ Kopecek and Libal see bifacial solar cells as the concept with the 'highest potential to increase the output power of PV systems at the lowest additional cost'. ${ }^{3}$ Indeed, the bifacial solar cell market has been gathering pace for a couple of years and

${ }^{a}$ Helmholtz-Zentrum Berlin für Materialien und Energie, Albert-Einstein-Straße 16, D-12489 Berlin, Germany. E-mail: christiane.becker@helmholtz-berlin.de

${ }^{b}$ Zuse Institute Berlin, Takustraße 7, D-14195 Berlin, Germany

$\dagger$ Electronic supplementary information (ESI) available. See DOI: 10.1039/c9se00750d

\$ These authors contributed equally to this work. several major PV companies, such as Sanyo, ${ }^{6}$ Yingli, $^{7}$ PVG solutions, bSolar/SolAround, ${ }^{8}$ and Trina Solar $^{9}$ introduced bifacial modules. The tenth edition of the International Technology Roadmap for Photovoltaics (ITRPV) predicts a global market share of more than 50\% for bifacial modules in $2029 .^{10}$ Large-scale bifacial PV power plants already have been realised and showed a higher energy yield than their monofacial counterparts. ${ }^{\mathbf{1 1}}$

The levelised cost of electricity (LCOE) is a very relevant economic metric of a solar power plant. ${ }^{12}$ The performance of bifacial solar modules is heavily affected by their surroundings, because they can accept light from almost every direction. Hence, a vast amount of parameters influence the resulting LCOE, for example the module and land costs, module distance and inclination angle, albedo of the ground, geographical position and the weather conditions at the location of the solar farm. Liang et al. recently identified comprehensive simulation models for energy yield analysis as one of the key enabling factors. ${ }^{4}$ As an example, we briefly discuss how only two free parameters - land cost and module distance - affect the resulting LCOE, which makes it challenging to identify the sweet spot yielding a minimum LCOE: if two rows of tilted solar modules are installed close to each other, many modules can be installed per area. However, at too small distances shadowing will limit the rear side irradiance and consequently the total 
energy yield. ${ }^{\mathbf{1 3 , 1 4}}$ In contrast, putting the rows of modules far apart from each other maximizes the irradiance at the rear side and the energy yield per module. The number of modules installed per area, however, is lower and the overall energy yield of the solar farm decreases. The module inclination angle is a third free parameter, closely connected to the two aforementioned module distance and land cost, and obviously affects shadowing of neighboring solar panel rows and hence energy yield and LCOE of a bifacial solar farm, too.

Historically, the module inclination angle was usually set to the geographical latitude of the solar farm location, and the module distance was either set to a fixed value based on experience $^{15}$ or to the minimum module distance without mutual shadowing on the day of winter solstice at $9 \mathrm{am}^{\mathbf{1 6}}$ or noon. ${ }^{17}$ However, it has turned out that these rule-of-thumb estimates often do not lead to a minimised LCOE. ${ }^{\mathbf{1 8}}$ One reason is that these models did not consider the cost of land. Recently Patel et al. considered land costs when optimising bifacial solar farms. ${ }^{16}$ However, also in this study the module distance and inclination angle were preset according to above mentioned winter solstice rule. Considering the enormous market growth of bifacial solar cell technology, finding the optimum configuration yielding minimum LCOE is highly desired. With the PV system costs in $\$$ per Watt peak (Wp), land costs in $\$$ per area and the geographic location of the solar farm as known input variables, inversely finding the optimal geometrical configuration of a bifacial PV field is a computational challenging multidimensional optimisation task.

In this study, we apply a multi-parameter Bayesian optimisation in order to minimise the LCOE of large-scale bifacial solar power plants. We present a comprehensive illumination model for bifacial solar arrays and calculate the annual energy yield (EY) based on TMY3 (Typical Meteorological Year 3) data for four exemplary locations near Seattle, Dallas, Mojave Desert and Havana. We calculate optimal module inclination angles and module distances yielding minimal LCOE for various module to land cost ratios. We find that our calculated optima strongly depend on both the module to land cost ratio and the geographical location. We conclude that currently used rule-ofthumb estimates for optimal module distance and tilting angle must be reconsidered. Our method enables the user to extract clear design guidelines for mono- and bifacial large-scale solar power plants principally anywhere on Earth.

\section{Illumination model}

With the illumination model we calculate the irradiance onto a solar module, which is placed somewhere in a big PV-field. We assume this field to be so big that effects from its boundaries can be neglected, but for smaller fields this might be a relevant effect caused by higher irradiance on the edges due to decreased self-shading. Further, we assume the modules to be homogeneous: we neglect effects from the module boundaries or module space in between the solar cells. Hence, we can treat this problem as 2-dimensional with periodic boundary conditions, as illustrated in Fig. 1. A similar approach was pursued for example by Marion et al. ${ }^{19}$ In the current model we assume the solar modules to be completely black, which means they do not reflect any light which could reach another module.

The PV field is irradiated from direct sunlight under the Direct Normal Irradiance (DNI) $\S$ and the direction $n_{\mathrm{S}}$, which is determined by the solar azimuth $\phi_{\mathrm{S}}$ and the solar zenith $\theta_{\mathrm{S}}$. The latter is connected to the solar altitude $a_{\mathrm{S}}$ (the height above the ground) via $a_{\mathrm{S}}=90^{\circ}-\theta_{\mathrm{S}}$. Further, the PV field receives diffuse light from the sky, which is given as Diffuse Horizontal Irradiance (DHI). However, for calculating the total irradiance onto the module, also light reflected from the ground and shadowing by the other modules must be taken into account.

Due to the typical geometry of a power plant the specular reflected DNI from the front side will seldom reach the back side of the front row. The diffuse reflectivity of the module should be significantly lower. In the current model we therefore assume the solar modules to be completely black and to not reflect any light. This might lead to a slight underestimation of the illumination.

Fig. 1 shows the different components of light, which can reach the front of a PV module at point $P_{\mathrm{m}}$. The numbers 1.-4. correspond to the numbers in the figure - illumination on the sky is w.l.o.g. indicated for module \#2 while illumination from the ground is indicated w.l.o.g. for module \#5.

(1) Direct sunlight hits the modules under the direction $n_{\mathrm{S}}$. It leads to the irradiance component $I_{\mathrm{dir}, \mathrm{f}}^{\mathrm{sky}}(s)=\mathrm{DNI} \cos \sigma_{\mathrm{ms}}$, where $s$ is the distance between the lower end of the module $B_{2}$ and $P_{\mathrm{m}}$, $s=\overline{B_{2} P_{\mathrm{m}}}$, and $\sigma_{\mathrm{ms}}$ is the angle between the module surface normal and the direct incident sunlight.

(2) Diffuse skylight $I_{\mathrm{diff}, \mathrm{f}}^{\text {sky }}(s)$ hits the module at $P_{\mathrm{m}}$ from directions within the wedge determined by $\Varangle D_{1} P_{\mathrm{m}} D_{2}$. Diffuse light does not only reach the module from directions within the $x z$-plane but from a spherical wedge, which is closely linked to the sky view factor as for example used by Calcabrini et al. ${ }^{20}$

(3) $I_{\text {dir, }}^{\text {gr. }}(s)$ denotes direct sunlight that hits the module after it was reflected from the ground.

(4) Finally, $I_{\text {diff,f }}^{\text {gr. }}(s)$ denotes diffuse skylight that hits the module after it was reflected from the ground.

All four components are summarized in Table 1 . Table 2 denotes all parameters that are used as input to the model.

The total irradiance (or intensity) on front is given by

$$
I_{\mathrm{f}}(s)=I_{\mathrm{dir}, \mathrm{f}}^{\mathrm{sky}}(s)+I_{\mathrm{diff}, \mathrm{f}}^{\mathrm{sky}}(s)+I_{\mathrm{dir}, \mathrm{f}}^{\mathrm{gr}}(s)+I_{\mathrm{diff}, \mathrm{f}}^{\mathrm{gr}}(s),
$$

and similar for the back side with a subscript $b$ instead of $f$. In total, we hence consider eight illumination components on our module.

As noted above, the incident light is given as DNI and DHI. The nonuniform irradiance distribution on the module front and back surfaces has to be considered.21,22 For the further treatment, it is therefore convenient to define unit-less geometrical distribution functions as for the components arising from direct sunlight and diffuse skylight, respectively. The geometrical distribution functions are closely related to the concept of view factors, which is often used for such illumination models. ${ }^{\mathbf{4 2 0 , 2 3}}$ Usually, view factors are defined such that

$\S$ The irradiance or intensity is the radiant power a surface receives per area. 


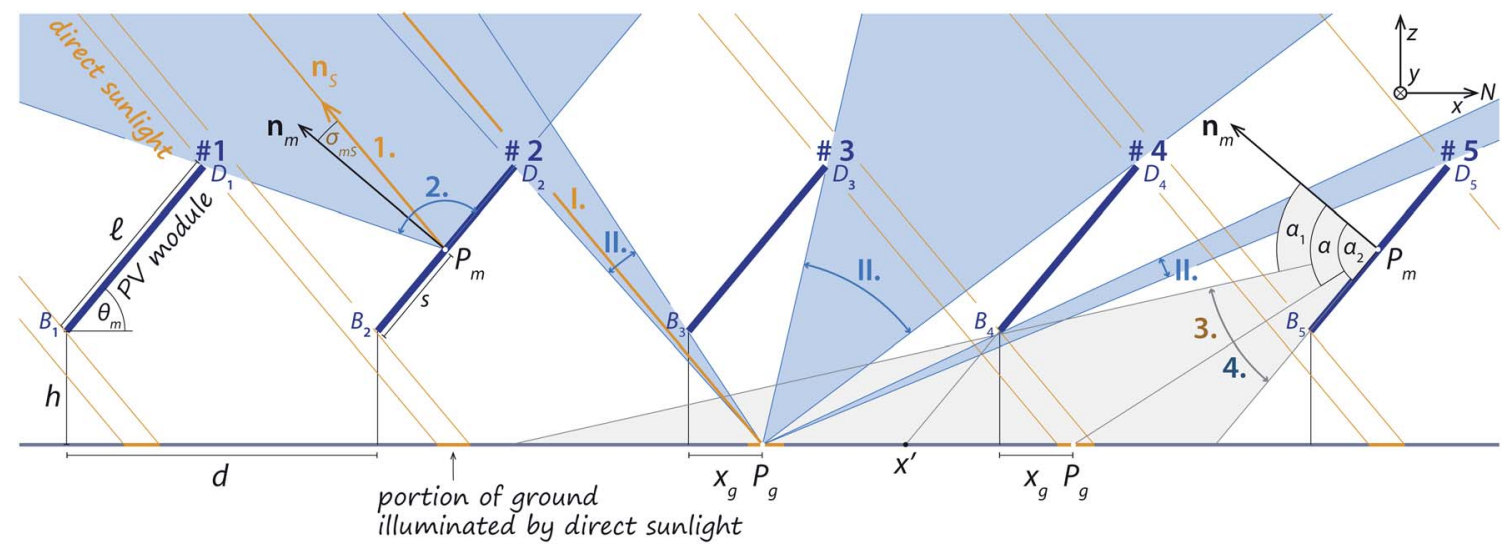

Fig. 1 Illustrating the geometrical configuration of a (periodic) PV field and the illumination components, which reach each module on the front. The modules are labeled with \#1-\#5. At \#1, the geometrical parameters $h, \ell, d$ and $\theta_{\mathrm{m}}$ are illustrated $-d$ is the horizontal length of a unit cell. At $\# 2$, the two irradiance components illuminating the module from the sky at $P_{m}$ are indicated: 1 . direct and 2. diffuse. Below \#3, the I. direct and II. diffuse illumination of point $P_{\mathrm{g}}$ on the ground are illustrated - here diffuse illumination origins from three angular intervals. On \#5 the angular range of light reaching $P_{m}$ from the ground is indicated. It consists of 3 . direct and 4 . diffuse light being reflected from the ground. Components 1.-4. are summarized in Table 1. Here, we assume w.l.o.g. that the PV system is located on the northern hemisphere and oriented towards South.

Table 1 The four irradiance components which constitute the illumination of a solar module in dependence of the position $P_{m}$ on the module as defined in Fig. 1 , where $s$ is the distance $\overline{B P_{m}}$. These components have to be considered for front and back sides - hence eight components in total. The numbers correspond to the numbers in Fig. 1

\begin{tabular}{llc}
\hline 1. & $\begin{array}{l}\text { Direct irradiance from the sky } \\
+ \text { circumsolar brightening }\end{array}$ & $I_{\text {dir }}^{\text {sky }}(s)$ \\
2. & $\begin{array}{l}\text { Diffuse irradiance from the sky } \\
\text { Diffuse irradiance from the } \\
\text { ground originating from direct sunlight } \\
+ \text { circumsolar brightening }\end{array}$ & $I_{\text {diff }}^{\text {sky }}(s)$ \\
& $\begin{array}{l}\text { Diff } \\
\text { Diffuse irradiance from the ground } \\
\text { originating from diffuse skylight }\end{array}$ & \\
& & $I_{\text {diff }}^{\text {gr. }}(s)$
\end{tabular}

they describe the radiation from one area onto another area, hence they give the average radiation onto the area, e.g. a module. However, we do not seek the mean irradiation on a module but the minimal irradiation. This is because of the electric properties of PV modules, as described in Section 3.1.

$$
\iota_{\mathrm{dir}, \mathrm{f}}(s):=\frac{I_{\mathrm{dir}, \mathrm{f}}(s)}{\mathrm{DNI}} \text { and } \iota_{\mathrm{diff}, \mathrm{f}}(s):=\frac{I_{\mathrm{diff}, \mathrm{f}}(s)}{\mathrm{DHI}}
$$

In eqn (2) we omitted the superscripts "sky" and "gr.". The calculation of the components $\iota_{\mathrm{dir}, \mathrm{f}}^{\mathrm{gr}}(s)$ and $\iota_{\mathrm{diff}, \mathrm{f}}^{\mathrm{gr}}(s)$ requires the integration over geometrical distribution functions on the ground $\gamma_{\mathrm{dir}}\left(x_{\mathrm{g}}\right)$ and $\gamma_{\mathrm{diff}}\left(x_{\mathrm{g}}\right)$, where $x_{\mathrm{g}}$ is the coordinate of the point $P_{\mathrm{g}}$ on the ground.

In particular, we have where we omitted the subscripts "diff" and "dir". The coordinate $x_{\mathrm{g}}(s, \alpha)$, on which $\gamma_{\text {dir }}$ and $\gamma_{\text {diff }}$ are evaluated, is defined such that the angle between the line $\overline{P_{g} P_{\mathrm{m}}}$ and the module normal $n_{\mathrm{m}}$ is equal to $\alpha$ - the integration parameter. In Fig. 1 the fractions of the ground, which are illuminated by direct sunlight, are marked in orange.
Table 2 The input parameters required to calculate the different parameters of the PV system

\begin{tabular}{ll}
\hline Module parameters (depicted in Fig. 1) \\
$\ell$ & Module length $(\mathrm{m})$ \\
$w$ & Module width $(\mathrm{m})$ \\
$d$ & Module spacing $(\mathrm{m})$ \\
$h$ & Module height above the ground $(\mathrm{m})$ \\
$\theta_{\mathrm{m}}$ & Module tilt angle \\
Solar parameters & \\
DNI & Direct normal irradiance $\left(\mathrm{W} \mathrm{m}^{-2}\right)^{a}$ \\
DHI & Diffuse horizontal irradiance $\left.(\mathrm{W} \mathrm{m})^{-2}\right)^{a}$ \\
$\theta_{\mathrm{S}}$ & Zenith angle of the sun \\
& (connected to solar altitude $a_{\mathrm{S}}$ via $a_{\mathrm{S}}=90^{\circ}-\theta_{\mathrm{S}}$ \\
$\phi_{\mathrm{S}}$ & Azimuth of the Sun \\
$A$ & Albedo of the ground
\end{tabular}

Economical parameters

$c_{\mathrm{P}} \quad$ Peak power related system costs (\$ per kWp)

$c_{\mathrm{L}} \quad$ Land consumption related costs $\left(\$\right.$ per $\left.\mathrm{m}^{2}\right)$

${ }^{a}$ This parameter also can be spectral. Then, the unit would be $\mathrm{W}\left(\mathrm{m}^{2}\right.$ $\mathrm{nm})^{-1}$.

$$
\iota_{\mathrm{f}}^{\mathrm{gr} .}(s)=\frac{A}{2} \int_{\alpha_{1}(s)}^{\alpha_{2}} \gamma\left[x_{\mathrm{g}}(s, \alpha)\right] \cos \alpha \mathrm{d} \alpha
$$

Fig. 2 shows an example for illumination onto the ground: subfigure (a) illustrates the position of the solar modules \#1 and $\# 2$. Subfigure (b) shows the geometrical distribution functions on the ground. $\gamma_{\text {diff }}$ is minimal below the module where the angle covered by the module is largest; and maximal at $x^{\prime}$, because here the ground sees least shadow from module \#1.

Depending on the geometrical module parameters and the position of the Sun, the directly illuminated area (1) may lay completely within the unit cell as in the examples in Fig. 1 and 


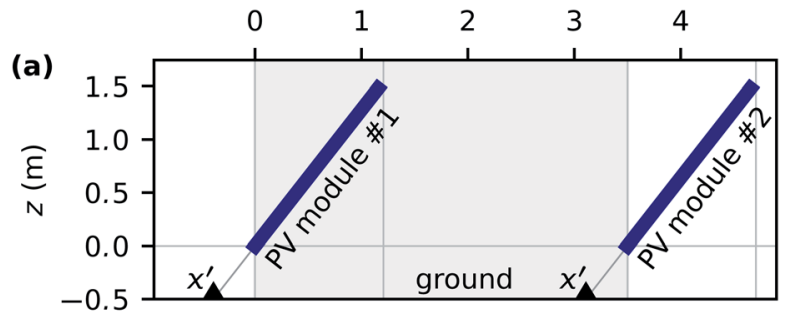

(b)

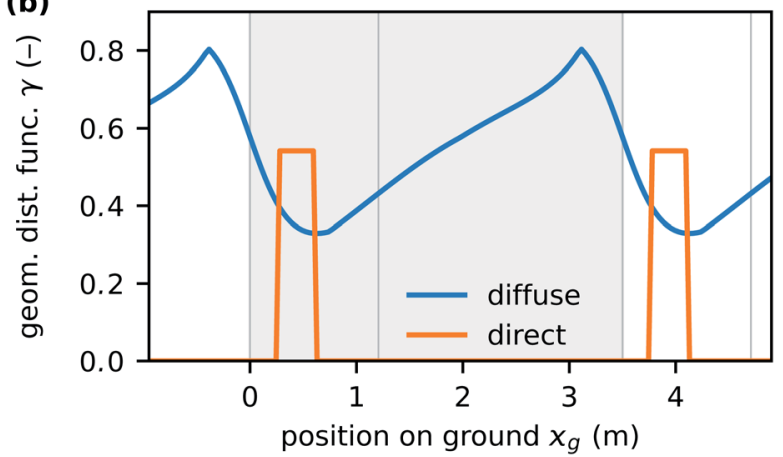

Fig. 2 An example for (a) a module configuration and (b) the corresponding diffuse and direct geometrical distribution functions at the ground $\gamma_{\text {diff }}$ and $\gamma_{\text {dir }}$. The following parameters were used: $\ell=1.96 \mathrm{~m}$, $d=3.50 \mathrm{~m}, h=0.50 \mathrm{~m}$ and $\theta_{\mathrm{m}}=52^{\circ}$. The solar position for the direct component was $\theta_{\mathrm{S}}=57.2^{\circ}$ and $\phi_{\mathrm{S}}=143.3^{\circ}$ (Berlin, 20 September 2019, 11:00 CEST). The unit cell is represented as shaded area.

2, (2) it may extend from one unit cell into the next or (3) no direct light can reach the ground. The latter can occur when the module spacing $d$ decreases or when the solar altitude $a_{\mathrm{S}}$ is low.

Fig. 3 shows the eight geometrical distribution functions $\iota$ corresponding to the irradiance components hitting the PV module on its front and back sides. While the functions originating from the sky (a) are stronger on the front side, the components originating from the ground (b) are stronger on the back side. This can be understood by the opening angles: the opening angle towards the sky is larger on the front side, but the opening angle of the ground is larger at the back.

All calculations presented in this work were performed with Python using numpy as numerical library for fast tensor operations.

\section{Annual energy yield}

\subsection{Calculating the energy yield}

We calculate the annual electrical energy yield EY by feeding the illumination model described in Section 2 with irradiance data. To demonstrate the features of the model, we use TMY3 (Typical Meteorological Year 3) data for this work. TMY3 data is well suited to estimate the solar energy yield for thousands of different locations. ${ }^{24}$ Amongst other parameters, the TMY3 data contain hourly $\mathrm{DHI}(t)$ and $\mathrm{DNI}(t)$ values. The overall EY given in $[\mathrm{EY}]=\mathrm{kW} \mathrm{h}$ per $\mathrm{m}^{2}$ and year is the sum of the energy yields harvested over the course of a year at the module front and back sides, $\mathrm{EY}=\mathrm{EY}_{\mathrm{f}}+\mathrm{EY}_{\mathrm{b}}$, which are calculated with
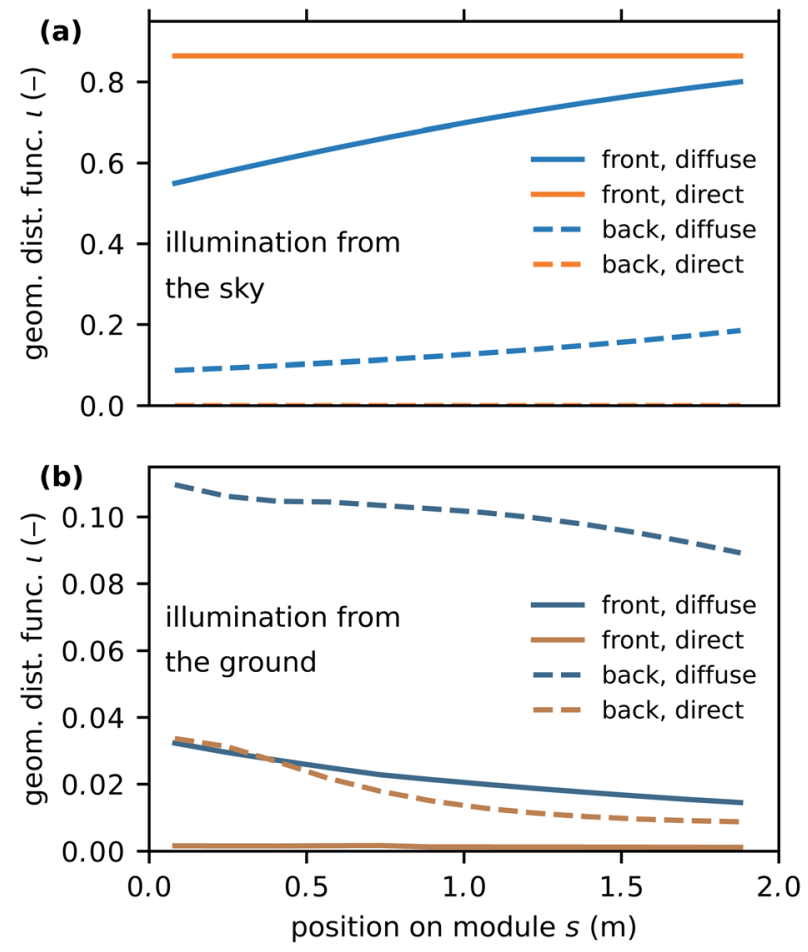

Fig. 3 Geometrical distribution functions on the module for light the module receives (a) from the sky and (b) the ground. The following parameters were used: $\ell=1.96 \mathrm{~m}, d=3.50 \mathrm{~m}, h=0.50 \mathrm{~m}, \theta_{\mathrm{m}}=52^{\circ}$, and albedo $A=30 \%$. The solar position for the direct components was $\theta_{\mathrm{S}}=57.2^{\circ}$ and $\phi_{\mathrm{S}}=143.3^{\circ}$ (Berlin, 20 September 2019, 11:00 CEST).

$$
\begin{gathered}
\mathrm{EY}_{\mathrm{f}}=\eta_{\mathrm{f}}\left\{\sum_{i}\left[\iota_{\mathrm{dir}, \mathrm{f}}^{\text {sky }}\left(\hat{s}_{i}, t_{i}\right)+\iota_{\mathrm{dir}, \mathrm{f}}^{\mathrm{gr}}\left(\hat{s}_{i}, t_{i}\right)\right] \operatorname{DNI}\left(t_{i}\right) \Delta t\right. \\
\left.+\sum_{i}\left[\iota_{\text {diff }, \mathrm{f}}^{\text {sky }}\left(\hat{s}_{i}\right)+\iota_{\text {diff }, \mathrm{f}}^{\text {gr. }}\left(\hat{s}_{i}\right)\right] \operatorname{DHI}\left(t_{i}\right) \Delta t\right\},
\end{gathered}
$$

and $\mathrm{EY}_{\mathrm{b}}$ with a subscript $\mathrm{b}$ instead of $\mathrm{f}$. TMY3 data is available at the time stamps $t_{i}$ and $\Delta t$ is the time between two time stamps, which is typically $1 \mathrm{~h}$ for TMY3 data. $\eta_{\mathrm{f}}$ and $\eta_{\mathrm{b}}$ denote the power conversion efficiency for light impinging on the front and back sides of the solar module, respectively. By setting $\eta_{\mathrm{f}}=\eta_{\mathrm{b}}=1$, eqn (4) delivers the annual radiant exposure on the front $H_{\mathrm{f}}$ (and similarly $H_{\mathrm{b}}$ ). As we aim at for an optimisation of the sitespecific geometry of a solar park in a general way and not only for a specific solar module type, we assume a constant mean efficiency with $\eta_{\mathrm{f}}=0.20$ and $\eta_{\mathrm{b}}=0.18$, and hence a bifaciality factor of $0.9 .^{5}$ The following optimisation results on solar panel arrays can therefore be understood as site-specific design guideline for any solar module type with efficiencies in this range. In reality, the power conversion efficiency is certainly not a constant but depends on multiple module specific factors such as module temperature, irradiance and angle of incidence. ${ }^{25,26}$

The $\iota$-functions are evaluated on the position $\hat{s}_{i} \in \mathbb{P}_{\mathrm{m}}$, where $\mathbb{P}_{\mathrm{m}}=\left\{s_{1}, s_{2}, \ldots, s_{N_{\mathrm{m}}}\right\}$ is the set of all considered positions along the module. In a conventional PV module, all cells are 

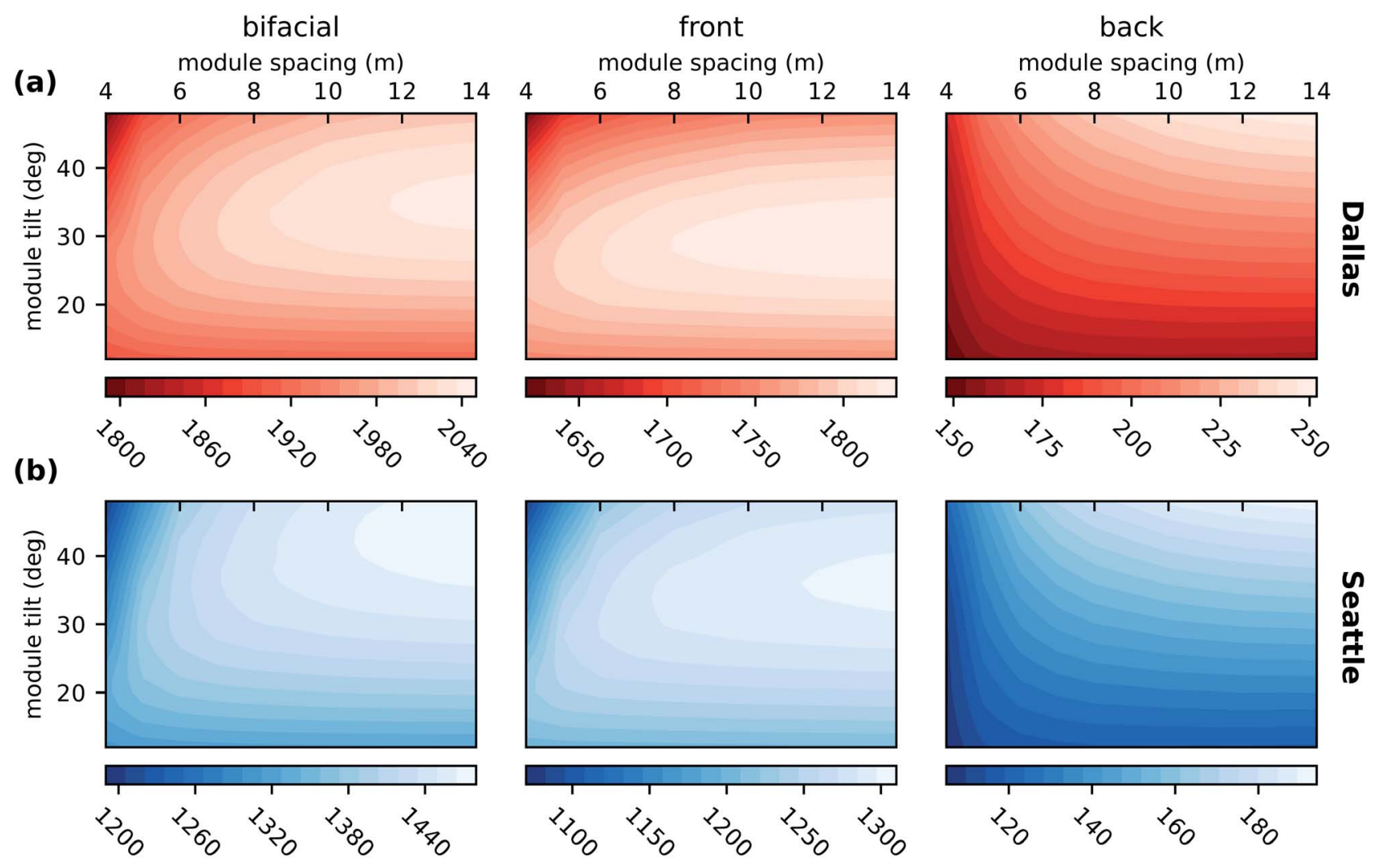

annual radiant exposure $(\mathrm{kWh})$

Fig. 4 Annual radiant exposure for bifacial modules and the contributions from front and back sides in a large PV field as a function of module spacing $d$ and module tilt $\theta_{\mathrm{m}}$. Results are shown for Dallas, TX, (top row) and Seattle, WA, (bottom row). The annual radiation yield is calculated using eqn (4) with $\eta_{\mathrm{f}}=\eta_{\mathrm{b}}=1$. Simulated with $\mathrm{m}$ module height $h=0.5 \mathrm{~m}$ and albedo $A=30 \%$.

electrically connected in series and therefore the cell generating the lowest current limits the overall module current. To take this into account, we determine $\hat{s}_{i}$ such that

$$
\left(I_{\mathrm{f}}+I_{\mathrm{b}}\right)\left(\hat{s}_{i}, t_{i}\right) \leq\left(I_{\mathrm{f}}+I_{\mathrm{b}}\right)\left(s, t_{i}\right)
$$

for all $s \in \mathbb{P}_{\mathrm{m}}$. This means that the position on the module with the lowest irradiance, which is proportional to the solar cell current, determines the overall module performance. For highend solar modules, the module performance might be higher depending on how bypass diodes are implemented. Therefore, our condition establishes a lower bound of the module performance under certain illumination conditions.

To model the diffuse irradiance we use the Perez model, that is widely used for solar cell simulations. The Perez model distinguishes three different components of diffuse irradiance to calculate the intensity on a tilted plane: isotropic dome, circumsolar brightening and horizontal brightening. For modelling the illumination the circumsolar brightening component is added to the direct normal irradiance because it is centred at the position of the sun. The horizontal brightening is shaded by rows in front and back and is therefore not considered to calculate the final irradiance. For the isotropic dome irradiation on the module, the corresponding geometrical distribution functions $\iota^{\text {diff }}(s)$ need to be calculated only once.

For the components arising from direct sunlight, also the geometrical distribution functions $\iota^{\operatorname{dir}}\left(s, t_{i}\right)$ are time-dependent, because they depend on the position of the $\operatorname{Sun}\left(\theta_{\mathrm{S}, \mathrm{i}}, \phi_{\mathrm{S}, \mathrm{i}}\right)$, which we calculate using the Python package Pysolar. ${ }^{28}$

\subsection{Results and discussion}

As an example, we discuss results for two locations with different climates: first, Dallas/Fort Worth area, Texas (TX), USA (Denton, $195 \mathrm{~m}$ elevation, $33.21^{\circ} \mathrm{N}, 97.13^{\circ} \mathrm{W}$ ) with a humid subtropical climate (Köppen-Geiger classification $\mathrm{Cfa}^{29}$ ) with hot, humid summers and cool winters. Secondly, Seattle, Washington (WA), USA (Boeing Field, $47.68^{\circ} \mathrm{N}, 122.25^{\circ} \mathrm{W}$ ) with a warm-temperate (Mediterranean) climate (Köppen-Geiger classification $\mathrm{Csb}^{29}$ ) with relatively dry summers and cool wet winters. Fig. $\mathrm{S} 1 \uparrow$ shows climate diagrams for these two locations.

In the ESI, $\uparrow$ we also show results for Daggett, USA (Mojave desert, $585 \mathrm{~m}$ elevation, $34.87^{\circ} \mathrm{N}, 116.78^{\circ} \mathrm{W}$ ) with a hot desert climate (Köppen-Geiger classification $\mathrm{BWh}^{29}$ ) and Havana,

I See for example ref. 27, appendix E. 
Cuba (Casa Blanca, $50 \mathrm{~m}$ elevation, 23.17 $\mathrm{N}, 82.35^{\circ} \mathrm{W}$ ) with a tropical climate (Köppen-Geiger classification $\mathrm{A}^{29}$ ).

Fig. 4 shows the annual radiant exposure in (a) Dallas and (b) Seattle for bifacial PV modules (left) in a big PV field and the contributions from the front (middle) and back sides (right). The data shown in the figure are calculated like the energy yield according to eqn (4), where we set $\eta_{\mathrm{f}}=\eta_{\mathrm{b}}=1$. We see that $H$ generally increases with the module spacing. However, it is not economical to have a too large distance between the rows as we will see when considering the electricity cost in Section 4 .

For Dallas, the optimal angle for monofacial modules, which only can utilize front illumination, is about $28^{\circ}$; it is mainly determined by direct sunlight. For back illumination, $H$ increases significantly with the module inclination angle $\theta_{\mathrm{m}}$ : hardly any direct light reaches the module at the back, but contributions from diffuse sky and reflected from the ground increase with $\theta_{\mathrm{m}}$. Increasing the module tilt further reduces the shaded area on the ground and therefore increases ground illumination. The optimal module tilt for a bifacial module is a compromise between the optimal tilt for the front and beneficial higher tilt angles for back contribution. Overall, the optimal module tilt for bifacial modules is significantly higher than for monofacial modules. Here it is about $36^{\circ}$.

Overall, the trends for Seattle are comparable to those for Dallas. However, we can identify differences: the overall radiant exposure is much lower because Seattle sees around 2170 annual Sun hours, compared to about $2850 \mathrm{~h}$ in Dallas. ${ }^{30}$ Further, the optimal tilt for monofacial and bifacial modules is $32^{\circ}$ and $44^{\circ}$, respectively, which is explained by the higher latitude of Seattle.

For the front side illumination we see the interesting effect that, while the latitude of Seattle and Dallas differ by $14.5^{\circ}$, the respective optimal tilt angles only differ by $4^{\circ}$. This is probably because of the higher contribution on the annual radiant exposure from the summer months in Seattle compared to Dallas. While in Seattle May to September contribute $77 \%$ of the annual radiant exposure this is only $65 \%$ in Dallas. Because the module irradiance during the summer months (with higher elevation angles of the Sun) benefits from lower tilt angle $\theta_{\mathrm{m}}$ values this can explain the difference of latitude to optimal tilt angles. The higher fraction of diffuse light in Seattle that also benefits the radiant exposure on the front side for small $\theta_{\mathrm{m}}$ might additionally increase this effect.

Fig. 5 shows how much the different irradiation components contribute to the annual radiant exposure for a bifacial module with $d=10 \mathrm{~m}$ module spacing, $\theta_{\mathrm{m}}=34^{\circ}$ tilt and albedo $A=30 \%$ in Dallas: about $74 \%$ of the total exposure arises from direct sunlight impinging onto the module front, $14 \%$ are due to diffuse skylight impinging onto the front but the fraction of light that reaches the front from the ground is almost negligible. However, of the $10.5 \%$ exposure received by the back, around $88 \%$ is reflected from the ground. Hence, the albedo only has little influence onto the energy yield of monofacial modules but is very relevant for bifacial modules. Fig. S4† shows corresponding results for Seattle. Compared to Dallas, Seattle shows $1.4 \%$ per larger contribution by the back side. While the front side receives radiation with a ratio of $3.5: 1$ of direct to diffuse light, for the back side, this ration is close to $1: 1$. These results show that four factors drive the gain of bifacial modules instead of monofacial modules: the albedo of the ground, the module tilt angle, the module spacing and the overall fraction of diffuse light.

Also the mounting height $h$ affects the bifacial gain. Increasing the mounting height monotonically raises the energy yield. Therefore it is difficult to optimise this parameter without knowing additional technical and commercial constraints. However, we find that the bifacial gain starts to saturate for a height above $0.5 \mathrm{~m}$, which is in agreement with work from Kreinin et al. ${ }^{17}$ Since a mounting height of $h=0.5 \mathrm{~m}$ seems realistic all simulations in our work are performed with this mounting height.

\section{Minimising the electricity cost}

In Section 3 we discussed how to calculate the annual electrical energy yield and we analysed how the annual radiant exposure on the modules depends on the module spacing and tilt for two examples: Dallas and Seattle. In this section, we are going to

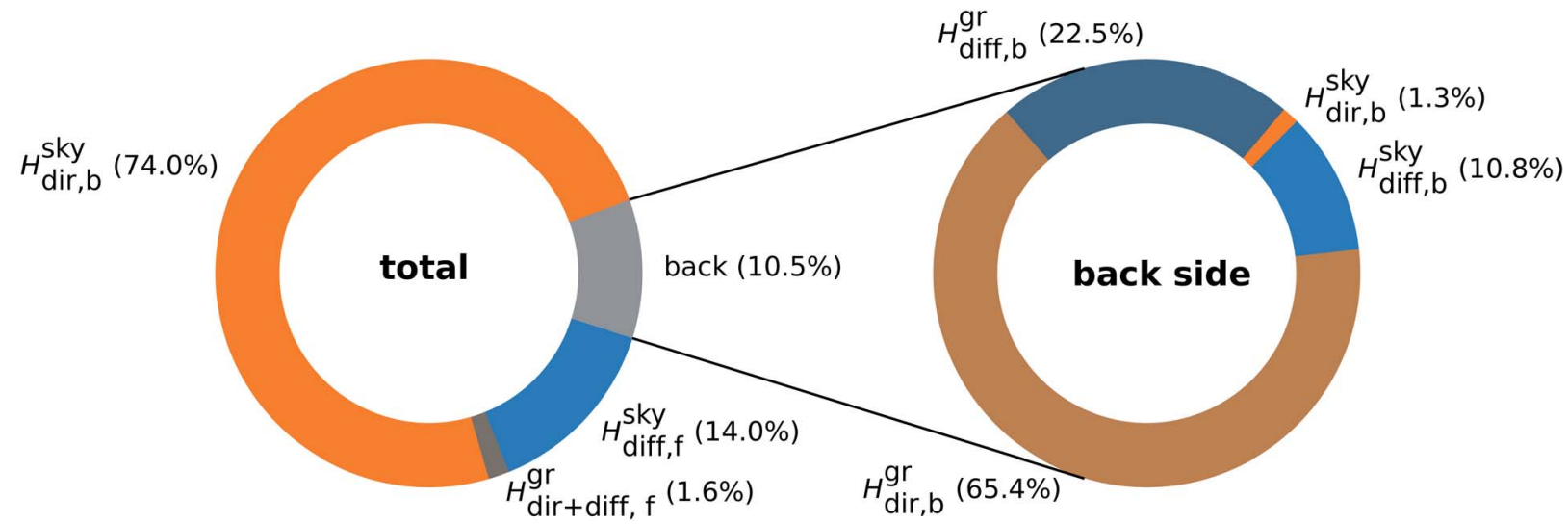

Fig. 5 (left) Different annual radiant exposure components for a bifacial solar cell in Dallas. (right) Detailed picture for the back side. Simulated with module spacing $d=10 \mathrm{~m}$, module tilt $\theta_{\mathrm{m}}=34^{\circ} \mathrm{m}$, module height $h=0.5 \mathrm{~m}$ and albedo $A=30 \%$. 
derive a simple model for the electricity cost and perform some cost optimisations.

\subsection{Levelised cost of electricity}

As a measure for the electricity cost we use the levelised cost of electricity (LCOE), which is a key metric for electricity generation facilities. In the simplest case, the LCOE is given as the total cost $C_{\mathrm{F}}$ spent in the facility during its lifetime $T$ (in years) divided by the total amount of electric energy $E_{\text {total }}$ generated in that time. Using the yield of a meteorological representative year allows to calculate the total yield by multiplying the annual power production with the lifetime of the facility.

$$
\mathrm{LCOE}=\frac{C_{\mathrm{F}}}{E_{\mathrm{total}}}=\frac{C_{\mathrm{F}}}{E_{\mathrm{F}} T},
$$

where $E_{\mathrm{F}}$ is the electric energy generated by the PV field in one year. In more involved models also costs of capital and discount rates are taken into account.\|

The total cost can be split into two components, associated with the peak power $C_{\mathrm{P}}$ (including modules, inverters, mounting etc.) and the land consumption $C_{\mathrm{L}}$ (lease, fences, cables etc.) of the facility.

$$
C_{\mathrm{F}}=C_{\mathrm{P}}+C_{\mathrm{L}}
$$

By considering a facility with a PV-field of $M$ rows with $N$ modules each the costs can be calculated per unit cell,

$$
C_{\mathrm{F}}=\left(C_{\mathrm{P}, \mathrm{m}}+C_{\mathrm{L}, \mathrm{m}}\right) M N
$$

The peak-power related costs per module $C_{\mathrm{P}, \mathrm{m}}$ are calculated with

$$
C_{\mathrm{P}, \mathrm{m}}=c_{\mathrm{P}} \eta_{\mathrm{f}} I_{\mathrm{P}} \ell w
$$

where $c_{\mathrm{P}}$ denotes the peak-power associated costs given in $\left[c_{\mathrm{p}}\right]=$ $\$$ per $\mathrm{kWp}$, which we use as input parameter. $I_{\mathrm{P}}=1 \mathrm{~kW} \mathrm{~m}^{-2}$ is the peak irradiance as used for standardized PV characteriza$\operatorname{tion}^{31}$ and $w$ and $\ell$ denote the module width and length, respectively. $\eta_{\mathrm{f}}$ denotes the power conversion efficiency on the front side of the solar cell.

The cost of land consumption per module depends on module width $w$ and spacing $d$,

$$
C_{\mathrm{L}, \mathrm{m}}=c_{\mathrm{L}} d w
$$

with the land $\operatorname{cost} c_{\mathrm{L}}$ given in $\left[c_{\mathrm{L}}\right]=\mathrm{S}$ per $\mathrm{m}^{2}$, which is an input parameter.

The annual generated electric energy of the PV field is given by with the annual yield EY according to eqn (4).

$$
E_{\mathrm{F}}=\mathrm{EY} \ell w M N
$$

Combining eqn (6)-(11) and simplifying leads to the expression which is independent of the field dimensions $M$ and $N$ and the module width $w$.

|| See for example ref. 27, chapter 21.

$$
\mathrm{LCOE}=\frac{\ell I_{\mathrm{P}} \eta_{\mathrm{f}} c_{\mathrm{P}}+d c_{\mathrm{L}}}{\ell \mathrm{EYT}}
$$

In this study, we assume for the overall costs of the PV system $c_{\mathrm{m}}=1000 \mathrm{\$}$ per $\mathrm{kWp}$, which includes all costs over the lifetime of the solar park, such as PV module investment, balance of system cost, planning, capital cost and others. The land cost is not included in this quantity. The lifetime is assumed to be $T=25$ years, a typical time span for the power warranty of solar cell modules. ${ }^{27}$

In our optimisation, we aim to minimize the LCOE as parameter of the module spacing $d$ and the solar module tilt $\theta_{\mathrm{m}}$. We perform the optimisation for five land-cost scenarios $c_{\mathrm{L}}$, in which we assume to include all costs that are related to an increase of area such as lease, cables, fences etc. Table 3 gives an overview of the cost scenarios and the resulting fraction of the land costs on the total costs, $\left(C_{\mathrm{L}} / C_{\mathrm{F}}\right)$.

\subsection{Optimisation method}

As optimisation method we use Bayesian optimisation, which is well suited to find a global minimum of black box functions, which are expensive to evaluate. ${ }^{32}$ Bayesian optimisation has been used in a wide variety of applications such as robotics, ${ }^{33}$ hyper parameter tuning ${ }^{34}$ or physical systems. ${ }^{35,36}$

In principle, Bayesian optimisation consists of two components: a surrogate model that approximates the black box function and its uncertainty (based on previously evaluated data points) and an acquisition function that determines the next query point from the surrogate model. After evaluating the function for the queried data point the surrogate model is updated and the next step can be computed with the acquisition function. This cycle is repeated until a specified number of steps or a convergences criteria is reached. We use the implementation from scikit-optimize with Gaussian process as surrogate model and expected improvement as acquisition function. ${ }^{37}$

\subsection{Optimisation results}

Traditionally, the optimal tilt and module spacing are often estimated with the winter solstice rule. ${ }^{38,39}$ The optimal distance between two rows of modules is defined as the shortest distance for which the shadow of a row of modules does not hit the next

Table 3 Overview of used cost scenarios. Right column shows the share of land consumption on total costs for different scenarios assuming row spacing $d=10 \mathrm{~m}$, module length $\ell=1.96 \mathrm{~m}$ and $\eta_{\mathrm{f}}=$ $20 \%$

$c_{\mathrm{P}}(\$$

\begin{tabular}{lll} 
per kWp $)$ & $c_{\mathrm{L}}\left(\$\right.$ per $\left.^{2}\right)$ & $C_{\mathrm{L}} / C_{\mathrm{F}}(\%)$ \\
\hline 1000 & 1.00 & 2.5 \\
1000 & 2.50 & 6.0 \\
1000 & 5.00 & 11.3 \\
1000 & 10.00 & 20.3 \\
1000 & 20.00 & 33.8
\end{tabular}


row of modules in a specified solar time window (e.g. 9 am-3 $\mathrm{pm}$ ) on winter solstice. As a rule of thumb the tilt is often chosen to be equivalent to the latitude of the facility location. However these rules do not consider the economic trade off between land costs and energy yield or typical weather patterns (e.g. foggy winters) that vary for different locations.

Fig. 6 and 7 shows the optimisation results for a field of (a) bifacial and (b) monofacial PV modules in Dallas and Seattle, respectively. Black dots mark evaluated data points, the red dot marks the found optimum and the color map shows the interpolation of the LCOE by the Gaussian process. The blue line indicates the winter solstice rule ( $9 \mathrm{am})$.

We see that the optimum shifts to smaller module spacing with increasing land cost. Further, also the optimal module tilt decreases in order to compensate for increased shadowing because of less module spacing. Overall, bifacial installations show larger module spacing and higher tilt angles in optimal configurations compared to monofacial technology. With increasing land costs and therefore reduced optimal module spacing the cost landscape gets increasingly steep. The sensitivity of the optimised parameters increases and using nonoptimal geometrical configurations results in increasing yield loss. Seattle shows the same trends for optimal configuration in different cost scenarios. Compared to Dallas optimal tilt and spacing are higher.
Our optimisation results differ significantly from the geometric parameters obtained from the winter solstice rule. For Dallas the winter solstice rule only provides comparable optimal parameters for $c_{\mathrm{L}}=5 \mathrm{~S}$ per $\mathrm{m}^{2}$. In Seattle, the optimal distances are shorter and the optimal module tilts are larger than expected from the winter solstice rule for all cost scenarios. This can be understood when considering the large share of diffuse light during the Seattle winter, which mitigates shading losses significantly.

Table 4 compares the LCOE obtained from optimisation to results for rule-of-thumb geometries (tilt angle = latitude, distance according to 9 am winter solstice rule) for different land cost scenarios. Depending on the location and cost scenario we see a reduction of LCOE of up to $23 \%$. The rule-ofthumb approach shows its weakness especially in Seattle. There is a general trend for higher reductions at high cost scenarios, where the cost landscape is increasingly steep (see Fig. 6 and 7). The optimisation for Havana in general exhibits the smallest reduction of LCOE but compared to the other locations there is no clear trend for higher reductions for higher land costs.

From these results it is clear that the winter solstice rule is not able to properly reflect different economic trade-offs or different illumination conditions over the course of the year. This is especially true when setting the tilt angle to the latitude of the location. For a minimal LCOE module tilt and spacing should be optimised independently from each other. Further,
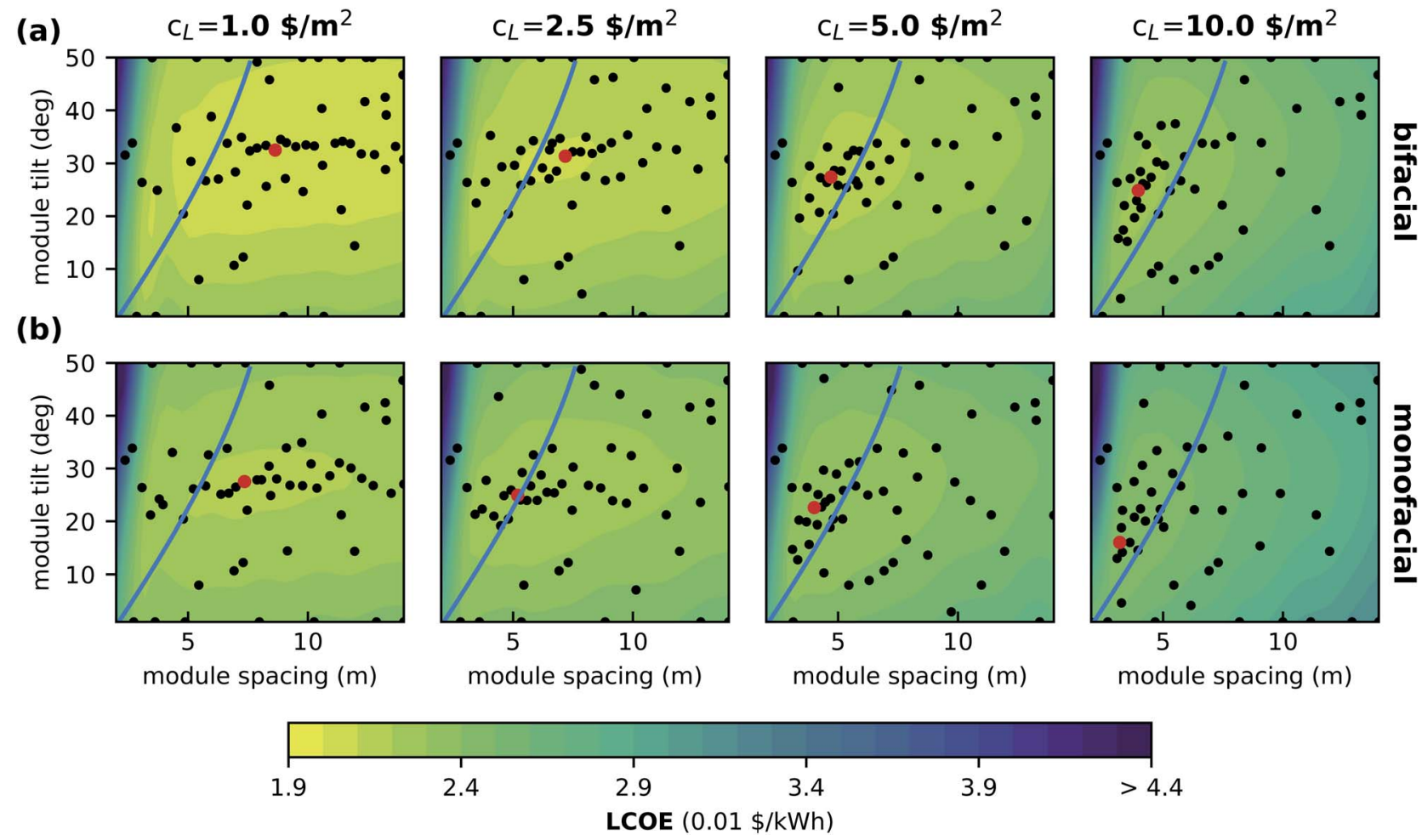

Fig. 6 Results of the Bayesian optimisation for minimising LCOE of (a) bifacial and (b) monofacial PV modules in Dallas with the land cost $C_{L}$ scenarios 1, 2.5, 5, and $10 \$$ per $\mathrm{m}^{2}$. Black dots mark evaluated configurations and the color map corresponds to the interpolation by a Gaussian process. The red dot indicates the minimal LCOE found by the optimisation. The blue curves indicate rule-of-thumb module distance according to 'no shadowing of neighboring modules at winter solstice'. Simulations with albedo $A=30 \%$, module height $h=0.5 \mathrm{~m}$ and peak power costs $c_{\mathrm{p}}$ $=1000 \$$ per $\mathrm{kW} \mathrm{h}$. 

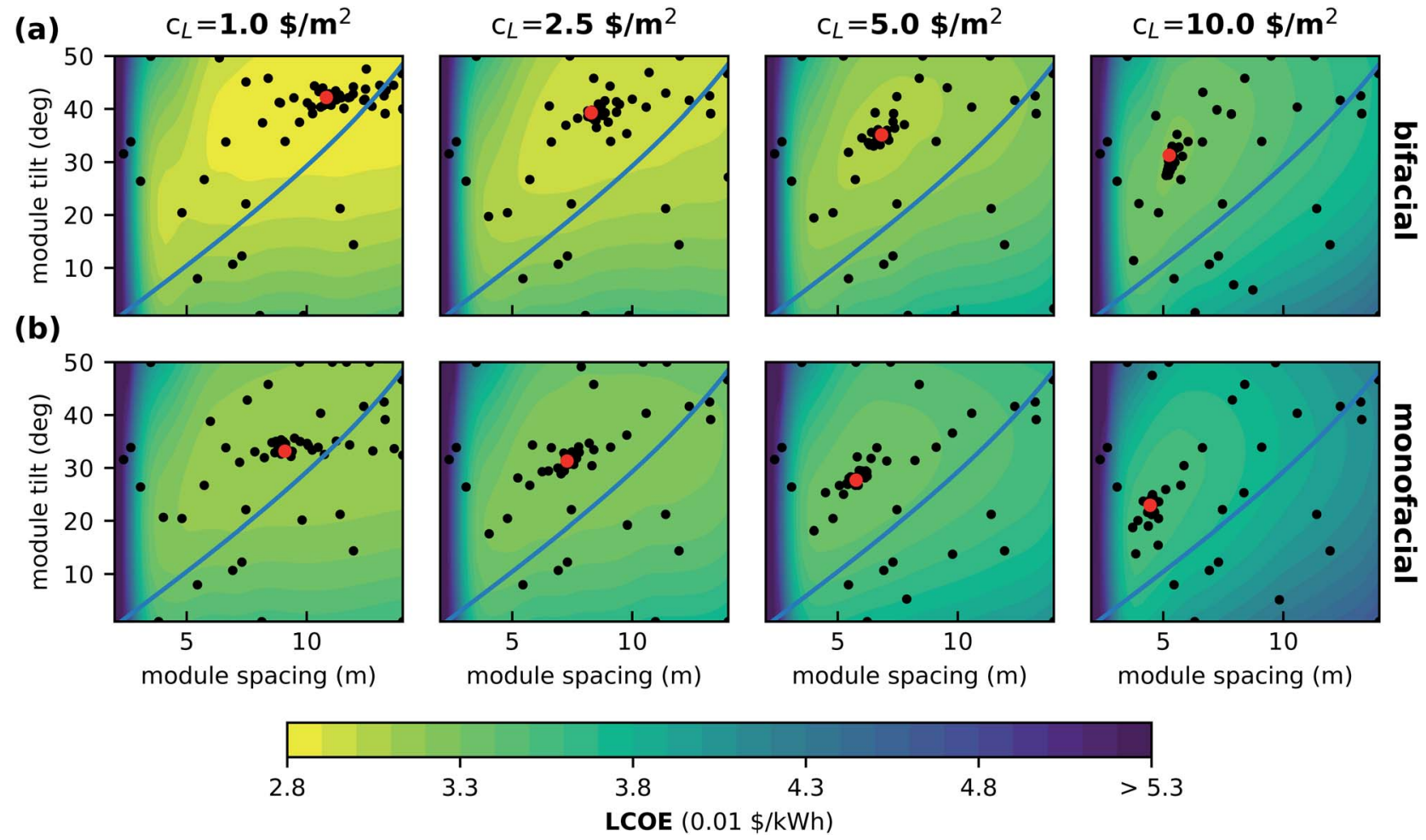

Fig. 7 Results of the Bayesian optimisation for minimising LCOE of (a) bifacial and (b) monofacial PV modules in Seattle with the land cost $C_{L}$ scenarios 1, 2.5, 5 and $10 \$$ per $\mathrm{m}^{2}$. Black dots mark evaluated configurations and the color map corresponds to the interpolation by a Gaussian process. The red dot indicates the minimal LCOE found by the optimisation. The blue curves indicate rule-of-thumb module distance according to 'no shadowing of neighboring modules at winter solstice'. Simulations with albedo $A=30 \%$, module height $h=0.5 \mathrm{~m}$ and peak power costs $c_{\mathrm{p}}$ $=1000 \$$ per $\mathrm{kW} \mathrm{h}$.

typical weather patterns and the local economic situation must be taken into account.

\subsection{Discussion}

The results of all optimisations are summarised in Fig. 8 and Table 5. We see that the optimal LCOE increases slightly with the land cost. Further, in Seattle the LCOE difference between mono- and bifacial modules is larger as in Dallas, Havana or the Mojave Desert. This is caused by the larger module tilt and diffuse light share in Seattle, which increases the fraction of illumination at the module back. Dallas and the Mojave Desert have comparable latitude but show a small difference in bifacial gain due to the higher diffuse light share in Dallas. As discussed above, the optimal module tilt decreases with increased land consumption cost $c_{\mathrm{L}}$.

In general, we see that for a utility scale solar cell plant both, the module tilt and the distance between rows, affect the annual energy yield. Increasing the distance increases the energy yield and the costs per module while tilt can be optimised costneutral. The optimal distance between rows is a compromise between increasing costs with higher land use for higher distances and lower energy yield due to shading for lower distances. This is true also for monofacial modules but due to the increased relevance of light reflected from the ground it is more relevant for bifacial modules.

Table 4 Comparing LCOE results for bifacial modules with optimised tilt and distance vs. rule-of-thumb parameters (module tilt equal latitude and distance according to 9 am winter solstice rule) for Dallas, Havana, Mojave and Seattle. Simulations with albedo $A=30 \%$, module height $h=$ $0.5 \mathrm{~m}$ and peak power costs $c_{\mathrm{P}}=1000 \$$ per $\mathrm{kW} \mathrm{h}$

\begin{tabular}{|c|c|c|c|c|c|c|c|c|c|c|c|c|}
\hline$c_{\mathrm{L}}\left(\$\right.$ per $\left.\mathrm{m}^{2}\right)$ & \multicolumn{4}{|c|}{ LCOE (cents) optimised } & \multicolumn{4}{|c|}{ Rule-of-thumb } & \multicolumn{4}{|c|}{ Reduction (\%) } \\
\hline 2.5 & 2.10 & 1.89 & 1.61 & 2.93 & 2.10 & 1.89 & 1.62 & 3.00 & 0.0 & 0.0 & 0.6 & 2.3 \\
\hline 5.0 & 2.17 & 1.94 & 1.67 & 3.07 & 2.18 & 1.94 & 1.68 & 3.24 & 0.5 & 0.0 & 0.6 & 5.2 \\
\hline 10.0 & 2.28 & 2.02 & 1.77 & 3.28 & 2.33 & 2.04 & 1.80 & 3.74 & 2.1 & 1.0 & 1.7 & 12.3 \\
\hline
\end{tabular}


(a)

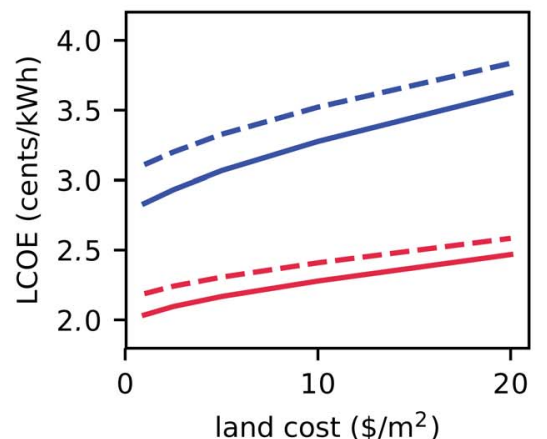

(b)

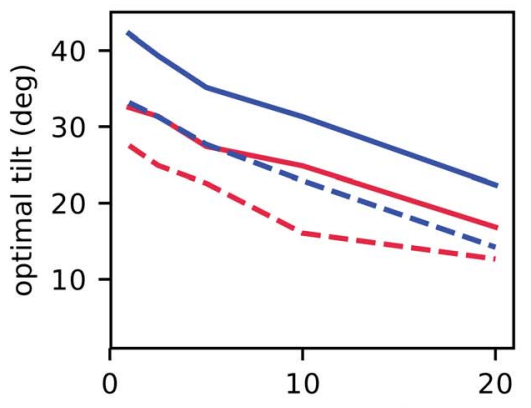

land cost $\left(\$ / \mathrm{m}^{2}\right)$ (c)

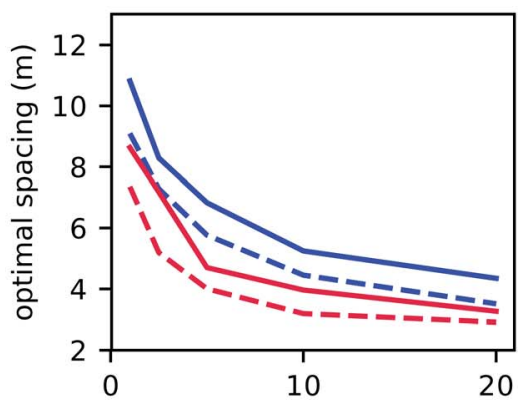

land cost $\left(\$ / \mathrm{m}^{2}\right)$

$\begin{array}{lll}\text { - Dallas bifacial } & \text { Seattle bifacial } \\ \text { - } & \text { Dallas monofacial } \quad \text { - } & \text { Seattle monofacial }\end{array}$

Fig. 8 Results of the optimisation for different land cost scenarios in Dallas (red lines) and Seattle (blue lines): (a) lowest LCOE and (b) optimal module tilt and (c) optimal spacing. Simulations with albedo $A=30 \%$, module height $h=0.5 \mathrm{~m}$ and peak power costs $c_{p} 1000 \$$ per $\mathrm{kW}$ h.

Table 5 Fraction of land cost $\left(C_{\mathrm{L}} / C_{\mathrm{F}}\right)$, module distance $d$ and bifacial gain for optimised configurations in different cost scenarios. Simulations with albedo $A=30 \%$, module height $h=0.5 \mathrm{~m}$ and peak power costs $C_{P}=1000 \$$ per $\mathrm{kW} \mathrm{h}$

\begin{tabular}{|c|c|c|c|c|c|c|c|c|c|c|c|c|}
\hline$c_{\mathrm{L}}\left(\$\right.$ per $\left.\mathrm{m}^{2}\right)$ & \multicolumn{4}{|c|}{$C_{\mathrm{L}} / C_{\mathrm{F}}(\%)$} & \multicolumn{4}{|l|}{$d(\mathrm{~m})$} & \multicolumn{4}{|c|}{ Bif. gain (\%) } \\
\hline 2.5 & 4.4 & 3.3 & 4.2 & 5.0 & 7.2 & 5.3 & 6.9 & 8.3 & 11.0 & 10.1 & 10.3 & 12.7 \\
\hline 5.0 & 5.7 & 4.7 & 6.1 & 8.0 & 4.7 & 3.9 & 5.1 & 6.8 & 9.6 & 9.0 & 9.4 & 11.7 \\
\hline 10.0 & 9.2 & 7.8 & 9.6 & 11.8 & 4.0 & 3.3 & 4.2 & 5.2 & 8.8 & 7.9 & 8.4 & 10.5 \\
\hline
\end{tabular}

The optimal configuration for bifacial solar cells depends on the radiation conditions and the albedo of the facility location. With increasing latitude (and therefore lower solar elevation angles), albedo and diffuse light contribution the bifacial gain will be increased and therefore make this type of PV technology more attractive for utility scale developers.

Cost optimisations for PV installation are quickly outdated because PV module prices have been decreasing for many years and land cost is very volatile. However the optimal installation geometry only depends on the ratio of land cost related to total costs and not absolute values. Hence, at a scenario of $c_{\mathrm{L}}=10 \$$ per $\mathrm{m}^{2}$ and $c_{\mathrm{P}}=1500 \mathrm{\$}$ per $\mathrm{kWp}$ yields the same optimisation result as $c_{\mathrm{L}}=5 \$$ per $\mathrm{m}^{2}$ and $c_{\mathrm{P}}=750 \mathrm{~S}$ per $\mathrm{kWp}$.

\section{Conclusions}

We developed a detailed model to calculate the irradiation onto both sides of a PV module, which is located in a large PV field. With this model, we could estimate the annual energy yield for monofacial and bifacial PV modules as a function of the module spacing and the module tilt. We assume a constant power conversion efficiency and a simple approach to calculate the levelised cost of electricity allowing for a technology independent modeling. Combined with a Bayesian optimisation algorithm, this allowed us to minimise the LCOE as a function of module spacing and module tilt for different land consumption costs. Due to the general approach the presented LCOE have the character of an example. It can be refined by implementation of module specific derating factors such as the temperature and incident angle dependent conversion efficiency behaviour.

Our results basically show that the bifacial gain and optimal geometry depend on the specific location and cost scenario. The bifacial gain can be expected to increase for locations with higher latitude and higher diffuse light share.

The usually used rule of thumb, no shadowing at wintersolstice and module tilt angle equal to the geographical latitude, leads to suboptimal module spacing and tilt combinations, because it does not account for economic trade-offs and the influence of the local climate. In contrast, optimising the parameters in Seattle can lead to a $23 \%$ reduction of LCOE for high land cost scenarios. This shows the significance of sitespecific and land-cost dependent optimisation and helps users to identify the configurations yielding minimal LCOE.

\section{Conflicts of interest}

There are no conflicts to declare.

\section{Acknowledgements}

We thank Lev Kreinin and Asher Karsenti from SolAround for fruitful discussions regarding the illumination model for 
bifacial solar cells. P. T. thanks the Helmholtz Einstein International Berlin Research School in Data Science (HEIBRiDS) for funding. The results were obtained at the Berlin Joint Lab for Optical Simulations for Energy Research (BerOSE) and the Helmholtz Excellence Cluster SOLARMATH of HelmholtzZentrum Berlin für Materialien und Energie, Zuse Institute Berlin and Freie Universität Berlin.

\section{Notes and references}

1 K. Yoshikawa, H. Kawasaki, W. Yoshida, T. Irie, K. Konishi, K. Nakano, T. Uto, D. Adachi, M. Kanematsu, H. Uzu and K. Yamamoto, Nat. Energy, 2017, 2, 17032.

2 A. Richter, M. Hermle and S. W. Glunz, IEEE J. Photovoltaics, 2013, 3, 1184-1191.

3 R. Kopecek and J. Libal, Nat. Energy, 2018, 3, 443-446.

4 T. S. Liang, M. Pravettoni, C. Deline, J. S. Stein, R. Kopecek, J. P. Singh, W. Luo, Y. Wang, A. G. Aberle and Y. S. Khoo, Energy Environ. Sci., 2019, 12, 116-148.

5 S. Chunduri and M. Schmela, Bifacial Solar Technology Report 2018 Edition, Taiyang news technical report, 2018.

6 Sanyo, Sanyo Canada launches first bifacial solar module -, 2009, https://www.greenlaunches.com/alternative-energy/ sanyo-canada-launches-first-bifacial-solar-module.php.

7 Yingli, Yingli's PANDA BIFACIAL Module Became the World's First Bifacial Module Certified by CGC, UL, and TUV Rheinland, 2018, http://ir.yinglisolar.com/news-releases/ news-release-details/yinglis-panda-bifacial-module-becameworlds-first-bifacial.

8 bSolar, bSolar launches High-Efficiency Bifacial Silicon Solar Cells, 2012, https:/www.photovoltaik.eu/article-44946330021/bsolar-launches-high-efficiency-bifacial-silicon-solarcells-.html.

9 TrinaSolar, Trina Solar to launch N-type i-TOPCon double-glass bifacial modules, 2019, https://solarpv.expert/2019/06/14/ trina-solar-to-launch-n-type-i-topcon-double-glass-bifacialmodules/.

10 ITRPV, 10th Edition of the International Technology Roadmap Photovoltaics, Vdma technical report, 2019.

$11 \mathrm{~N}$. Ishikawa and S. Nishiyama, presented at the 3rd Bifi PV Workshop, Miyazaki, Japan, 2016.

12 F. Fertig, S. Nold, N. Wöhrle, J. Greulich, I. Hädrich, K. Krauß, M. Mittag, D. Biro, S. Rein and R. Preu, Prog. Photovoltaics Res. Appl., 2016, 24, 800-817.

13 J. Appelbaum, Renewable Energy, 2016, 85, 338-343.

14 M. R. Khan, E. Sakr, X. Sun, P. Bermel and M. A. Alam, Appl. Energy, 2019, 241, 592-598.

15 I. Shoukry, J. Libal, R. Kopecek, E. Wefringhaus and J. Werner, Energy Procedia, 2016, 92, 600-608.

16 M. T. Patel, M. R. Khan, X. Sun and M. A. Alam, Appl. Energy, 2019, 247, 467-479.

17 L. Kreinin, A. Karsenty, D. Grobgeld and N. Eisenberg, 2016 IEEE 43rd Photovoltaic Specialists Conference (PVSC), 2016, pp. 2688-2691.

$18 \mathrm{P}$. Grana, The new rules for latitude and solar system design, 2018, https:/www.solarpowerworldonline.com/2018/08/ new-rules-for-latitude-and-solar-system-design/.
19 B. Marion, S. MacAlpine, C. Deline, A. Asgharzadeh, F. Toor, D. Riley, J. Stein and C. Hansen, 2017 IEEE 44th Photovoltaic Specialist Conference (PVSC), 2017.

20 A. Calcabrini, H. Ziar, O. Isabella and M. Zeman, Nat. Energy, 2019, 4, 206-215.

21 U. A. Yusufoglu, T. M. Pletzer, L. J. Koduvelikulathu, C. Comparotto, R. Kopecek and H. Kurz, IEEE J. Photovoltaics, 2015, 5, 320-328.

22 L. Kreinin, N. Bordin, A. Karsenty, A. Drori, D. Grobgeld and N. Eisenberg, 2010 35th IEEE Photovoltaic Specialists Conference, 2010, pp. 002171-002175.

23 X. Sun, M. R. Khan, C. Deline and M. A. Alam, Appl. Energy, 2018, 212, 1601-1610.

24 S. Wilcox and W. Marion, Users manual for TMY3 data sets, National Renewable Energy Laboratory Technical Report NREL/TP-581-43156, National Renewable Energy Laboratory Golden, CO, 2008.

25 N. Martín and J. M. Ruiz, Prog. Photovoltaics Res. Appl., 2005, 13, 75-84.

26 M. Jazayeri, S. Uysal and K. Jazayeri, 2013 High Capacity Optical Networks and Emerging/Enabling Technologies, 2013, pp. 44-50.

27 A. H. M. Smets, K. Jäger, O. Isabella, R. A. C. M. M. van Swaaij and M. Zeman, Solar energy: The physics and engineering of photovoltaic conversion technologies and systems, UIT Cambridge, 2016.

28 B. Stafford, Pysolar, 2018, DOI: 10.5281/zenodo.1461066.

29 M. Kottek, J. Grieser, C. Beck, B. Rudolf and F. Rubel, Meteorol. Z., 2006, 15, 259-263.

30 L. Ozborn, Average Annual Sunshine in American Cities, 2019, https:/www.currentresults.com/Weather/US/averageannual-sunshine-by-city.php.

31 ISO 9845-1:1992, International organization for standardization technical report, 1992.

32 B. Shahriari, K. Swersky, Z. Wang, R. P. Adams and N. de Freitas, Proc. IEEE, 2016, 104, 148-175.

33 A. Cully, J. Clune, D. Tarapore and J.-B. Mouret, Nature, 2015, 521, 503-507.

34 J. Snoek, H. Larochelle and R. P. Adams, Practical Bayesian Optimization of Machine Learning Algorithms, 2012, http:// papers.nips.cc/paper/4522-practical-bayesian-optimization.

35 P.-I. Schneider, X. G. Santiago, C. Rockstuhl and S. Burger, Proc. SPIE, 2017, 103350, 1033500.

36 H. C. Herbol, W. Hu, P. Frazier, P. Clancy and M. Poloczek, npj Comput. Mater., 2018, 4, 51.

37 T. Head, MechCoder, G. Louppe, I. Shcherbatyi, Fcharras, Z. Vinícius, Cmmalone, C. Schröder, Nel215, N. Campos, T. Young, S. Cereda, T. Fan, Rene-Rex, Kejia (KJ) Shi, J. Schwabedal, Carlosdanielcsantos, Hvass-Labs, M. Pak, F. Callaway, L. Estève, L. Besson, M. Cherti, K. Pfannschmidt, F. Linzberger, C. Cauet, A. Gut, A. Mueller and A. Fabisch, Scikit-Optimize/Scikit-Optimize: V0.5.2, 2018, DOI: 10.5281/zenodo.1207017.

38 M. T. Patel, M. R. Khan, X. Sun and M. A. Alam, Appl. Energy, 2019, 247, 467-479.

39 S. Sánchez-Carbajal and P. M. Rodrigo, Int. J. Photoenergy, 2019, 2019, 1-14. 